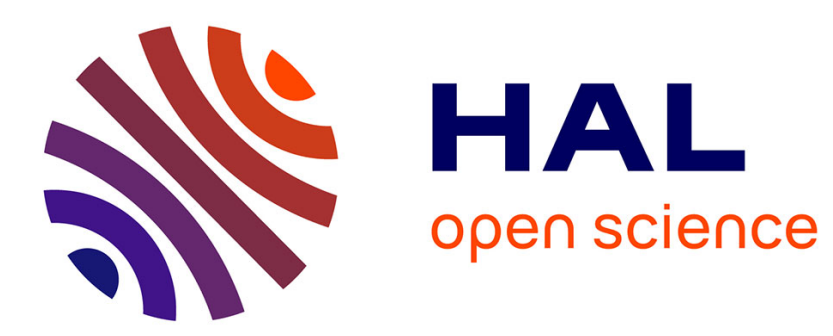

\title{
Light scattering in nematic liquid crystals in the presence of shear flow
}

\author{
H. Pleiner, H. Brand
}

\section{To cite this version:}

H. Pleiner, H. Brand. Light scattering in nematic liquid crystals in the presence of shear flow. Journal de Physique Lettres, 1983, 44 (1), pp.23-31. 10.1051/jphyslet:0198300440102300 . jpa-00232138

\section{HAL Id: jpa-00232138 https://hal.science/jpa-00232138}

Submitted on 1 Jan 1983

HAL is a multi-disciplinary open access archive for the deposit and dissemination of scientific research documents, whether they are published or not. The documents may come from teaching and research institutions in France or abroad, or from public or private research centers.
L'archive ouverte pluridisciplinaire HAL, est destinée au dépôt et à la diffusion de documents scientifiques de niveau recherche, publiés ou non, émanant des établissements d'enseignement et de recherche français ou étrangers, des laboratoires publics ou privés. 
Classification

Physics Abstracts

$05.70 \mathrm{~L}-05.40-61.30 \mathrm{G}$

\title{
Light scattering in nematic liquid crystals in the presence of shear flow
}

\author{
H. Pleiner \\ Universität Essen, D 4300 Essen, W. Germany
}

and H. Brand $\left(^{*}\right)$

Bell Tel. Laboratories, Murray Hill, N.J. 07974, U.S.A.

(Reçu le 31 septembre 1982, accepté le 9 novembre 1982)

\begin{abstract}
Résumé. - Nous examinons des fluctuations dans un cristal liquide nématique autour d'un état stationnaire de non-équilibre résultant d'un mouvement de cisaillement. Dans le cas particulier d'un vecteur d'onde situé dans le plan de cisaillement nous trouvons que le pic central du spectre de diffusion de la lumière (dû aux fluctuations du directeur), est élargi et aplati (en plus d'un déplacement Doppler trivial) et qu'il est réduit en intensité totale par comparaison avec l'état d'équilibre. Pour l'essentiel, les contributions de non-équilibre ne sont pas sensibles au vecteur d'onde puisque le mouvement de cisaillement rompt de l'extérieur une symétrie déjà spontanément brisée dans des nématiques.
\end{abstract}

\begin{abstract}
We theoretically investigate fluctuations in a nematic liquid crystal about a non-equilibrium steady state due to a stationary shear flow. For the special case, where the wave-vector lies in the shear plane, we find that the central peak in the light scattering spectrum (caused by director fluctuations) is (in addition to the trivial Doppler shift) considerably flattened, broadened and reduced in total intensity, if compared to the equilibrium situation. The non-equilibrium contributions are essentially wave-vector independent, because the shear flow breaks just that symmetry (externally), which is already broken spontaneously in nematics.
\end{abstract}

1. Introduction and results. - During the last several years there has been considerable interest in the study of light scattering in non-equilibrium (but near equilibrium) situations (Refs. [1-4] and references cited therein). All these studies focussed on the effects of fluctuations of simple fluids in non-equilibrium situations like e.g. static temperature gradient or stationary shear flow. Parts of the results of the theoretical papers were verified experimentally [5].

In a recent paper by the present authors [6] the influence of a static temperature gradient on the light scattering spectrum in nematic liquid crystals was investigated. The central peak caused by director fluctuations was found to be deformed into an asymmetric shape (and slightly shifted).

$\left(^{*}\right)$ Supported in part by Deutsche Forschungsgemeinschaft. 
Thereby the non-equilibrium contributions are wave-vector dependent and are measurable only by small angle scattering.

In the present paper we investigate the fluctuation spectrum in nematics when the non-equilibrium situation is due to a stationary shear flow. This is the unique case where the external force breaks just that symmetry, which is already spontaneously broken. That means, the director $\mathbf{n}$ of the nematic phase takes a certain direction in the shear plane (given by the flow alignment angle [7]) and fluctuations of $\mathbf{n}$ experience a restoring force, which does not vanish in the long wave length limit $k \rightarrow 0$. These are the fluctuations which give the non-equilibrium contributions to the central peak (caused by director fluctuations) in the light scattering spectrum of nematics. We find the equilibrium Lorentzian to be Doppler shifted, flattened, broadened in a symmetric but nonLorentzian manner, and reduced in total intensity because of the non-equilibrium situation. This reduction of total intensity stems from an increase of the elastic energy due to the external shear flow. The effects are large, roughly in the range of $10 \%$, and should, therefore be experimentally detectable.

Our results are obtained by the following procedure : the nonlinear hydrodynamic equations (including Langevin forces) are linearized about the non-equilibrium steady state. A fluctuationdissipation theorem is employed relating correlation functions of Langevin forces to hydrodynamic transport parameters. This relation is the same as in the case of true equilibrium, while the non-equilibrium situation is manifest in the linearized hydrodynamic equations, only (Section 2).

In order to be concise, we restrict ourselves to the special case, where the wave vector lies in the shear plane. Director fluctuations normal to the shear plane then couple only to the appropriate component of linear momentum and correlation functions of the former can easily be extracted from the linearized hydrodynamic equations (Section 3).

Implications for the ligth scattering spectrum are discussed subsequently.

2. Hydrodynamic equations. - We consider a nematic layer of thickness $d$ subject to a stationary shear flow. The geometry is sketched in figure 1 . In the steady state the director $\mathbf{n}^{0}=\hat{e}_{y} \cos \theta_{0}+$ $\hat{e}_{z} \sin \theta_{0}$ lies in the $y$-z-plane enclosing an angle $\theta_{0}\left({ }^{1}\right)$ with the $y$-axis and the velocity field is given by

$$
\mathbf{v}=\left(S_{0} \frac{d}{2}+S_{1} z\right) \hat{e}_{y} .
$$

This form contains e.g. the special case i) upper plate moving with velocity $v$ and lower plate fixed for $S_{0}=S_{1}=v / d$ and ii) upper and lower plate moving with velocity $v$ and $-v$, respectively for $S_{0}=0$ and $S_{1}=v / d$.

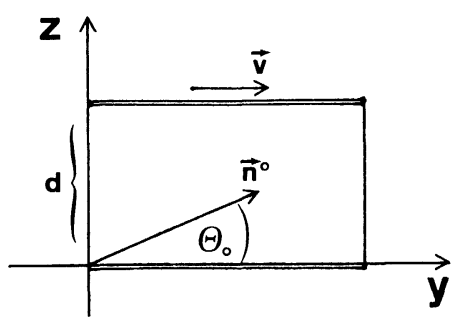

Fig. 1. - Scheme of the experimental set up : one or both plates are moved in $y$ and/or in $-y$-direction. The director field $\hat{n}^{0}$ encloses an angle $\theta_{0}$ with the velocity field $\mathbf{v}$. All calculations are done for a layer infinitely extended in the $x$ - and $y$-direction.

( $\left.{ }^{1}\right)$ This flow alignment angle $\theta_{0}$ can be expressed by the hydrodynamic flow parameter $\lambda$ [7] : $\cos 2 \theta_{0}=\lambda^{-1}$. 
In addition to equation (2.1) we have

$$
T=T_{0}, p=p_{0}, \rho=\rho_{0}, g_{x}=g_{z}=0, g_{y}=\rho_{0} v_{y}, \phi_{i j}=0
$$

where $p$ is the pressure, $\rho$ the density, $T$ the temperature, $g_{i}$ the density of linear momentum, and $\phi_{i j}$ is the thermodynamic conjugate of $\nabla_{i} n_{j}[8,9]$.

The equations of state are $[8,9]$

$$
\begin{aligned}
g_{i} & =\rho v_{i} \\
\delta \rho & =\chi_{\mathrm{E}} \delta p+\alpha \delta T \\
\phi_{i j} & =K_{i j k l} \nabla_{l} n_{k} .
\end{aligned}
$$

In appendix I we derive from the general nonlinear hydrodynamic equations (I . 1)-(I.3) including fluctuating forces [10] the equations linearized about the non-equilibrium steady state (I.4) and (I.5). As it is easily checked, $\delta n_{x}$ and $g_{x}$ decouple from all other variables in the special case $k_{x}=0$ (i.e. $\mathbf{k}$ lies in the shear plane). This simplifies the resulting formulas considerably and we, therefore, restrict ourselves to this case in the following.

Using the notations

$$
\begin{aligned}
& \nabla_{\|}=\hat{n}^{0} \cdot \nabla=\nabla_{y} \cos \theta_{0}+\nabla_{z} \sin \theta_{0} \\
& \nabla_{\perp}=\hat{e}_{x} \cdot\left(\nabla \times \hat{n}^{0}\right)=\nabla_{y} \sin \theta_{0}-\nabla_{z} \cos \theta_{0}
\end{aligned}
$$

we obtain from (I.4) and (I.5) the linearized equations for $\delta n_{x}$ and $g_{x}$ in the case $k_{x}=0$

$$
\begin{aligned}
\delta \dot{n}_{x}+\left(S_{0} \frac{d}{z}+S_{1} z\right) \nabla_{y} n_{x}-\frac{1}{2}(\lambda+1) \nabla_{\|} v_{x}-\xi\left(K_{2} \nabla_{\perp}^{2}\right. & \left.+K_{3} \nabla_{\|}^{2}\right) n_{x}+ \\
& +S_{1} \lambda \cos \theta_{0} \sin \theta_{0} \delta n_{x}=\pi x
\end{aligned}
$$

and

$$
\begin{aligned}
\dot{v}_{x} & +\left(S_{0} \frac{d}{z}+S_{1} z\right) \nabla_{y} v_{x}+\frac{1}{2 \rho_{0}}(\lambda+1) \nabla_{\|}\left(K_{2} \nabla_{\perp}^{2}+K_{3} \nabla_{\|}^{2}\right) n_{x}-\frac{1}{\rho_{0}}\left(v_{3} \nabla_{\|}^{2}+v_{2} \nabla_{\perp}^{2}\right) v_{x}+ \\
& +\frac{1}{\rho_{0}} S_{1}\left(2\left[v_{1}-v_{3}\right] \sin \theta_{0} \cos \theta_{0} \nabla_{\|}+\left[v_{3}-v_{2}\right]\left[\sin ^{2} \theta_{0}-\cos ^{2} \theta_{0}\right] \nabla_{\perp}\right) n_{x}=\nabla_{j} \sigma_{x j} .
\end{aligned}
$$

In equation (2.6) we have assumed incompressibility i.e. $v_{2}=v_{4}$ and $v_{5}=0$. For the general case [9]. Equations (2.5) and (2.6) differ from the hydrodynamic equations linearized about the equilibrium state by the occurrence of the $S_{0}$ and $S_{1}$-terms. $\pi_{x}$ and $\dot{\sigma}_{x j}$ are fluctuating forces [10] required to ensure the self-consistency of the hydrodynamic equations. They are assumed to be Gaussian and Markoffian, i.e. $f_{\alpha} \in\left\{\sigma_{x j}, \pi_{x}\right\}$

$$
\left\langle f_{\alpha}(1) f_{\beta}(2)\right\rangle=k_{\mathrm{B}}\left(\gamma_{\alpha \beta}+\gamma_{\beta \alpha}\right) \delta(1-2) \text {. }
$$

Restricting ourselves to situations near equilibrium, (i.e. well below any threshold of a shear flow instability [11-16]) the fluctuation dissipation theorem holds and the entropy production serves as a potential $[3,17]$. The coefficients $\gamma_{\alpha \beta}$ in equation $(2.7)$ are then given by the dissipative transport parameters of the appropriate hydrodynamic equations and equation (2.7) reads explicitly

$$
\begin{aligned}
\left\langle\sigma_{x j}\left(\mathbf{r}_{1} t_{1}\right) \sigma_{x l}\left(\mathbf{r}_{2} t_{2}\right)\right\rangle & =2 k_{\mathrm{B}} T v_{x j x l} \delta\left(\mathbf{r}_{1}-\mathbf{r}_{2}\right) \delta\left(t_{1}-t_{2}\right) \\
\left\langle\pi_{x}\left(\mathbf{r}_{1} t\right) \pi_{x}\left(\mathbf{r}_{2} t_{2}\right)\right\rangle & =2 k_{\mathrm{B}} T \xi \delta\left(\mathbf{r}_{1}-\mathbf{r}_{2}\right) \delta\left(t_{1}-t_{2}\right)
\end{aligned}
$$


Equations (2.8) are the same as in equilibrium and well known in literature [10]. The terms proportional to $S_{0}$ in equations $(2.5)$ and (2.6) can be treated exactly. They simply describe a Doppler shift, and they can be eliminated by a Galilean transformation to a proper moving frame. The $S_{1}$-terms reflect the non-equilibrium situation. Since their influence is expected to be rather small we will treat them perturbatively, i.e. we will only consider linear order in $S_{1}$. In appendix II it is shown that in this order the non-autonomous contributions proportional to $S_{1} z$ (in the drift terms) do not contribute to the correlation function of $\delta n_{x}$ and can, therefore, be dropped from equations (2.5) and (2.6) for our purposes $\left({ }^{2}\right)$.

We can now Fourier transform equations (2.5) and (2.6) and obtain the final equations

$$
\begin{aligned}
& \left(i \omega+S_{0} \frac{d}{2} i k_{y}+\hat{\mu}^{2}\right) v_{x}(k \omega)-\left(\frac{\chi+1}{2 \rho_{0}} i k_{\|} \hat{K}^{2}+i \hat{\alpha} S_{1}\right) \delta n_{x}(k \omega)=\frac{i}{\rho_{0}}\left(k_{\perp} \sigma_{x \perp}+k_{\|} \sigma_{x \|}\right) \\
& \left(i \omega+S_{0} \frac{d}{2} i k_{y}+\xi \hat{K}^{2}+\frac{1}{2} \Gamma\right) \delta n_{x}(k \omega)-i k_{\|} \frac{\chi+1}{2} v_{x}\left(k_{1} \omega\right)=\pi_{x}
\end{aligned}
$$

where we have introduced the transverse relaxation time

$$
\Gamma^{-1}=\left(2 S_{1} \lambda \cos \theta_{0} \sin \theta_{0}\right)^{-1}=S_{1}^{-1} \operatorname{cotan} 2 \theta_{0}
$$

and the abbreviations

$$
\begin{aligned}
& \hat{\mu}^{2}=\frac{1}{\rho_{0}}\left(v_{3} k_{\|}^{2}+v_{2} k_{\perp}^{2}\right) \equiv \mu_{3} k_{\|}^{2}+\mu_{2} k_{\perp}^{2} \\
& \hat{K}^{2}=K_{2} k_{\perp}^{2}+K_{3} k_{\|}^{2} \\
& \hat{\alpha}=2\left(\mu_{1}-\mu_{3}\right) \sin \theta_{0} \cos \theta_{0} k_{\|}+\left(\mu_{3}-\mu_{2}\right)\left(\sin ^{2} \theta_{0}-\cos ^{2} \theta_{0}\right) k_{\perp} .
\end{aligned}
$$

3. Correlation functions and light scattering. - For the correlation function $\left(^{3}\right) \chi_{n_{x} n_{x}}^{\omega}(\mathbf{k})$ we obtain from equations (2.8) and (2.9)

$$
\chi_{n_{x} n_{x}}^{\omega}(\mathbf{k})=2 k_{\mathrm{B}} T_{0} \frac{\xi\left(\Omega^{2}+\hat{\mu}^{2} \hat{\mu}^{2}\right)+\bar{\lambda}^{2} k_{\|}^{2} \hat{\mu}^{2}}{\left(-\Omega^{2}+\hat{\lambda}^{4}+\hat{\mu}^{2} \hat{\xi}^{2}\right)^{2}+\Omega^{2}\left(\hat{\mu}^{2}+\xi^{2}\right)^{2}}
$$

where

$$
\begin{aligned}
& \Omega=\omega+\frac{1}{2} S_{0} d k_{y} \\
& \hat{\xi}^{2}=\xi \hat{K}^{2}+\frac{1}{2} \Gamma \\
& \hat{\lambda}^{4}=\bar{\lambda}^{2} k_{\|}^{2} \hat{K}^{2}+\frac{1}{2}(\lambda+1) \rho_{0}^{-1} k_{\|} \hat{\alpha} S_{1}
\end{aligned}
$$

$\bar{\lambda}^{2}=\left(4 \rho_{0}\right)^{-1}(\lambda+1)$ and $\hat{\mu}^{2}, \hat{K}^{2}, \hat{\alpha}$ and $\Gamma$ are defined below equation (2.9).

It is then quite easy to show that (3.1) can be rewritten as the sum of two simple Lorentzians centred around $\Omega=0$

$$
\chi_{n_{x} n_{x}}^{\omega}(\mathbf{k})=2 k_{\mathrm{B}} T_{0}\left(\frac{M}{\Omega^{2}+\Omega_{1}^{2}}+\frac{P}{\Omega^{2}+\Omega_{2}^{2}}\right)
$$

( $\left.{ }^{2}\right)$ A similar situation arises for the density correlation function in simple fluids [3].

$\left({ }^{3}\right)$ The definition is

$$
\left\langle\delta n_{x}(\mathbf{k} \omega) \delta n_{x}^{*}\left(\mathbf{k}^{\prime} \omega^{\prime}\right)\right\rangle \equiv \chi_{n_{x} n_{x}}^{\omega}(\mathbf{k}) \delta\left(\omega-\omega^{\prime}\right) \delta\left(\mathbf{k}-\mathbf{k}^{\prime}\right)
$$


where

$$
\begin{aligned}
& M=\frac{\xi \hat{\mu}^{2} \hat{\mu}^{2}+\bar{\lambda}^{2} k_{\|}^{2} \hat{\mu}^{2}-\xi \Omega_{1}^{2}}{\Omega_{2}^{2}-\Omega_{1}^{2}} \\
& P=\frac{\xi \Omega_{2}^{2}-\xi \hat{\mu}^{2} \hat{\mu}^{2}-\bar{\lambda}^{2} k_{\|}^{2} \hat{\mu}^{2}}{\Omega_{2}^{2}-\Omega_{1}^{2}}
\end{aligned}
$$

and

$$
\Omega_{1,2}=\frac{i}{2}\left(\hat{\mu}^{2}+\hat{\xi}^{2}\right) \pm \frac{1}{2}\left[\left(\hat{\mu}^{2}+\hat{\xi}^{2}\right)^{2}+4 \hat{\lambda}^{4}+4 \hat{\mu}^{2} \hat{\xi}^{2}\right]^{1 / 2}
$$

The equilibrium part of $\chi_{n_{x} n_{x}}^{\omega}$ is obtained from (3.3)-(3.5) if one puts $S_{0}=0=S_{1}$ i.e. $\Omega=\omega$, $\hat{\xi}^{2}=\xi \hat{K}^{2}$ and $\hat{\lambda}^{4}=\bar{\lambda}^{2} k_{\|}^{2} K^{2}$, there.

The correlation function $\chi_{n_{x} n_{x}}^{\omega}(\mathbf{k})$ is measured by inelastic, depolarized light scattering [8, 9]. In our case the polarization of the electric vector changes from the direction parallel to $\hat{n}$ to the $x$-direction and $\omega$ and $\mathbf{k}$ are the differences between incoming and scattered frequencies and wave vectors, respectively.

Concentrating on the narrow and high peak in (3.3), which is responsible for the high scattering intensity in nematic liquid crystals, equation (3.3) simplifies into

$$
\chi_{n_{x} n_{x}}^{\omega}(\mathbf{k})=2 k_{\mathrm{B}} T_{0} P\left(\Omega^{2}+\Omega_{2}^{2}\right)^{-1} .
$$

Assuming the inequality $\hat{\mu}^{2} \gg \xi \hat{K}^{2}$ to hold, which implies also $\hat{\mu}^{2} \gg \hat{\xi}^{2}$ and $\hat{\mu}^{2} \hat{\mu}^{2} \gg \hat{\lambda}^{4}$ for all realistic valves of $S_{1}$, the equilibrium part of $\chi_{n_{x} n_{x}}^{\omega}(\mathbf{k})$ reads (cf. also [9])

$$
\chi_{n_{n^{n} n_{x}, \mathrm{eq}}}^{\omega}(\mathbf{k})=\frac{2 k_{\mathrm{B}} T_{0}}{\hat{K}^{2}} \cdot \frac{\omega_{1 / 2}}{\Omega^{2}+\omega_{1 / 2}^{2}}
$$

with

$$
\omega_{1 / 2}=\xi \hat{K}^{2}+\bar{\lambda}^{2} k_{\|}^{2} \hat{K}^{2} / \hat{\mu}^{2}
$$

where we have already accounted for a Doppler shift $\Omega \neq \omega\left(\right.$ for $S_{0} \neq 0$ ).

Up to linear order in $S_{1}$ the non-equilibrium part of

$$
\chi_{n_{x} n_{x}}^{\omega}(\mathbf{k}), \quad \delta \chi_{n_{x} n_{x}}^{\omega}(\mathbf{k}) \equiv \chi_{n_{x} n_{x}}^{\omega}(\mathbf{k})-\chi_{n_{x} n_{x}, \mathrm{eq}}^{\omega}(\mathbf{k})
$$

is given by

$$
\delta \chi_{n_{x} n_{x}}^{\omega}(\mathbf{k})=-\tilde{S}_{1} \frac{\omega_{1 / 2}}{\Omega^{2}+\omega_{1 / 2}^{2}} \chi_{n_{x} n_{x}, \mathrm{eq}}^{\omega}
$$

with

$$
\begin{aligned}
\tilde{S}_{1}= & \Gamma+(\lambda+1) \hat{\alpha} S_{1} k_{\|} / \hat{\mu}^{2} \\
= & 2 \lambda S_{1} \cos \theta_{0} \sin \theta_{0}+(\lambda+1) S_{1}\left(\left[\mu_{1}-\mu_{3}\right] \sin 2 \theta_{0} k_{\|}^{2}+\left[\mu_{2}-\mu_{3}\right] \cos 2 \theta_{0} k_{\|} k_{\perp}\right) \times \\
\times\left(\mu_{3} k_{\|}^{2}+\mu_{2} k_{\perp}^{2}\right)^{-1} & \\
= & S_{1} \tan 2 \theta_{0}+S_{1}\left(1+\cos 2 \theta_{0}\right)\left(\left[\mu_{1}-\mu_{3}\right] \tan 2 \theta_{0} k_{\|}^{2}+\left[\mu_{2}-\mu_{3}\right] k_{\|} k_{\perp}\right) \times \\
& \times\left(\mu_{3} k_{\|}^{2}+\mu_{2} k_{\perp}^{2}\right)^{-1}
\end{aligned}
$$

where $\omega_{1 / 2}$ and $\chi_{\text {eq }}$ are defined by (3.7). Thus, we have found that the(Doppler shifted) equilibrium Lorentzian is reduced in height by

$$
\frac{|\delta \chi(\Omega=0)|}{\chi_{\mathrm{eq}}(\Omega=0)}=\frac{\tilde{S}_{1}}{\omega_{1 / 2}} \equiv a
$$


and symmetrically broadened into a non-Lorentzian shape (Fig. 2). The relative increase in half-width is (up to linear order in $\widetilde{S}_{1}$ )

$$
\frac{\Omega\left(\chi=\frac{1}{2} \chi^{\mathrm{max}}\right)-\Omega\left(\chi_{\mathrm{eq}}=\frac{1}{2} \chi_{\mathrm{eq}}^{\mathrm{max}}\right)}{\Omega\left(\chi_{\mathrm{eq}}=\frac{1}{2} \chi_{\mathrm{eq}}^{\max }\right)} \approx \frac{1}{2} \frac{\tilde{S}_{1}}{\omega_{1 / 2}}=\frac{a}{z} .
$$

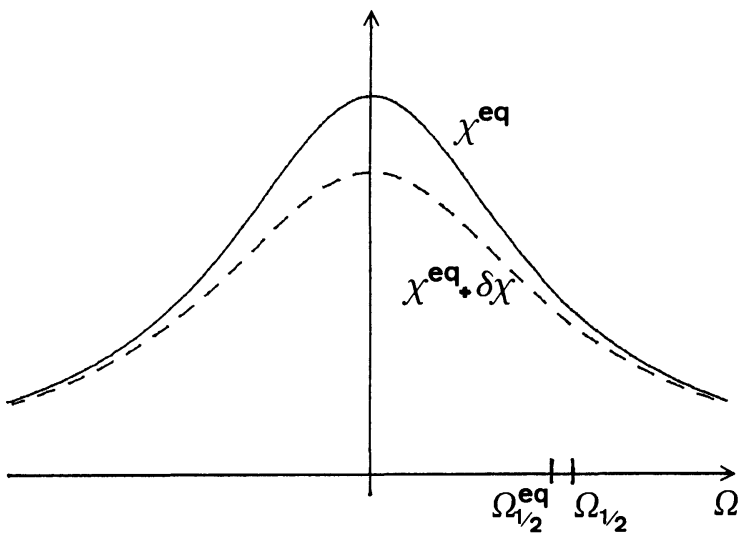

Fig. 2. - The central light-scattering peak (caused by transverse director fluctuations) in equilibrium $\chi^{\text {eq }}$ and non-equilibrium $\chi=\chi^{\mathrm{eq}}+\delta \chi$ according to equations (3.8) and (3.7) for typical values given in the text. $\Omega_{1 / 2}^{\text {eq }}$ and $\Omega_{1 / 2}$ are the frequencies, where the equilibrium and non-equilibrium peak take half of their maximal value, respectively.

As already discussed in the introduction, neither $\delta \chi$ nor $\tilde{S}_{1}$ vanish in the limit $k_{\|} \rightarrow 0$ (or $k_{\perp} \rightarrow 0$ ) and $\tilde{S}_{1}$ depends only very weak on the direction of $\mathbf{k}$. There, we have also pointed out the differences to the case of an external static temperature gradient [6].

Besides the above mentioned change in height and shape of the equilibrium peak, there is a reduction in overall intensity due to the non-equilibrium situation. For the static correlation function

$$
\chi_{n_{x} n_{x}}^{\text {stat }}(\mathbf{k}) \equiv \frac{1}{2 \pi} \int_{-\infty}^{+\infty} \chi_{n_{x} n_{x}}^{\omega}(\mathbf{k}) \mathrm{d} \omega
$$

we obtain from (3.6)

$$
\chi_{n_{x} n_{x}}^{\mathrm{stat}}(\mathbf{k}) \approx \frac{k_{\mathrm{B}} T_{0}}{\hat{K}^{2}} \frac{\omega_{1 / 2}^{-}}{\omega_{1 / 2}+\tilde{S}_{1}} \approx \chi_{n_{x} n_{x}, \mathrm{eq}}^{\mathrm{stat}}(\mathbf{k})(1-a) .
$$

This means that the elastic energy of the director fluctuations (i.e. the gradient energy) is raised and described by "effective static susceptibilities " $K_{2,3}^{\text {eff }}=K_{2,3}(1+a)$. This stabilization of the director field by the shear flow reduces the scattering intensity. Such an effect on the intensity is not present in the case of an external temperature gradient [6]. In order to get an estimate for the importance of the non-equilibrium effects we insert typical values $\left(k \approx 10^{3} \mathrm{~cm}^{-1}\right.$, $\xi=10 \mathrm{~cm} \mathrm{~s} \mathrm{~g}^{-1}, K \approx 10^{-6} \mathrm{~g} \mathrm{~cm} \mathrm{~s}^{-2}, \mu \approx 10^{-1} \mathrm{~cm}^{2} \mathrm{~s}^{-1}, \tan 2 \theta_{0} \approx 1,\left(\mu_{1}-\mu_{3}\right) / \mu_{3} \approx$ $\left.\left(\mu_{2}-\mu_{3}\right) / \mu_{2} \approx 10^{-1}, S_{1} \approx 1 \mathrm{~s}^{-1}, v \approx 1 \mathrm{~cm} \mathrm{~s}^{-1}, d=1 \mathrm{~cm}\right)$ and obtain $a \approx 10 \%$, which 
brings all above mentioned effects into the experimentally detectable range. For $S_{0}=S_{1}$ the Doppler shift is then $500 \mathrm{~Hz}$. The non-equilibrium effects on the light scattering spectrum predicted above are much bigger than any other non-equilibrium effects in other systems reported so far. The reasons are that in the present case i) the external force breaks just that symmetry which is spontaneously broken by the system and ii) fluctuations of that variable, which describes that broken symmetry, are the main sources for light scattering.

Appendix I. - For the nonlinear hydrodynamic equations of a nematic liquid crystal we have $[8,18]$

$$
\dot{\rho}+\nabla_{i} g_{i}=0
$$

$\dot{g}_{i}+\nabla_{k} v_{k} g_{i}+\nabla_{i} p+\frac{1}{2} \lambda \nabla_{j}\left(n_{i} \nabla_{l} \phi_{j l}+n_{j} \nabla_{l} \phi_{i l}\right)+\frac{1}{2} \nabla_{j}\left(\phi_{k j} \nabla_{i} n_{k}+\phi_{k i} \nabla_{j} n_{k}\right)-$

$$
-\frac{1}{2} \nabla_{j} \nabla_{k}\left(n_{i} \phi_{j k}-n_{j} \phi_{i k}\right)=\nabla_{j} v_{i j k l} \nabla_{k} v_{l}
$$

$\dot{n}_{i}+v_{k} \nabla_{k} n_{i}-\frac{1}{2} \lambda\left(\delta_{i l}-n_{i} n_{l}\right) n_{k}\left(\nabla_{l} v_{k}+\nabla_{k} v_{l}\right)+\frac{1}{2}\left(\delta_{i l}-n_{i} n_{l}\right) n_{k}\left(\nabla_{l} v_{k}-\nabla_{k} v_{l}\right)=\xi \nabla_{j} \phi_{i j}$

Linearizing equations (I.1)-(I.3) around the stationary state (2.1), (2.2), characterized by superscript zero, yields

$$
\begin{aligned}
& \delta \dot{\rho}+v_{i}^{0} \nabla_{i} \rho+\rho_{0} \nabla_{i} v_{i}=0=\left(\nabla_{j} v_{i j k l}\right)\left(\nabla_{k} v_{l}^{0}\right)+v_{i j k l}^{0} \nabla_{j} \nabla_{k} v_{l} \quad(\mathrm{I} \\
& \rho_{0} \dot{v}_{i}+v_{k}^{0} \rho_{0} \nabla_{k} v_{i}+\nabla_{i} p+\frac{1}{2} \lambda\left(n_{i}^{0} \nabla_{j} \nabla_{l} \phi_{j l}+n_{j}^{0} \nabla_{l} \nabla_{j} \phi_{i l}\right)-\frac{1}{2}\left(n_{i}^{0} \nabla_{j} \nabla_{k} \phi_{j k}-n_{j}^{0} \nabla_{j} \nabla_{k} \phi_{i k}\right)= \\
& \delta \dot{n}_{i}+v_{k}^{0} \nabla_{k} \delta n_{i}+\frac{1}{2} \lambda\left(n_{l}^{0} \delta n_{i}+n_{i}^{0} \delta n_{l}\right) n_{k}^{0}\left(\nabla_{l} v_{k}^{0}+\nabla_{k} v_{l}^{0}\right) \\
&+\frac{1}{2}\left(n_{l}^{0} \delta n_{i}+n_{i}^{0} \delta n_{l}\right) n_{k}^{0}\left(\nabla_{k} v_{l}^{0}-\nabla_{l} v_{k}^{0}\right) \\
&-\frac{\lambda}{2}\left(\delta_{i l}-n_{i}^{0} n_{l}^{0}\right) \delta n_{k}\left(\nabla_{l} v_{k}^{0}+\nabla_{k} v_{l}^{0}\right) \\
&+\frac{1}{2}\left(\delta_{i l}-n_{i}^{0} n_{l}^{0}\right) \delta n_{k}\left(\nabla_{l} v_{k}^{0}-\nabla_{k} v_{l}^{0}\right) \\
&+\frac{\lambda}{2}\left(\delta_{i l}-n_{i}^{0} n_{l}^{0}\right) n_{k}^{0}\left(\nabla_{l} v_{k}+\nabla_{k} v_{l}\right) \\
&+\frac{1}{2}\left(\delta_{i l}-n_{i}^{0} n_{l}^{0}\right) n_{k}^{0}\left(\nabla_{l} v_{k}-\nabla_{k} v_{l}\right)=\xi \nabla_{j} \phi_{i j}
\end{aligned}
$$

In equation (I.4) the expressions $\phi_{i j}$ and $v_{i j k l}$ are to be specified (cf. e.g. [8]). However, we need only $\nabla_{j} \phi_{x j}, \nabla_{j} v_{x j z y}$ and $v_{x j k l}^{0} \nabla_{j} \nabla_{k} v_{l}$ (cf. equation (2.1)) which are given by

$$
\begin{aligned}
\nabla_{j} \phi_{x j} & =K_{2} \varepsilon_{x j p} n_{p}^{0} \varepsilon_{k l q} n_{q}^{0} \nabla_{j} \nabla_{l} n_{k}+K_{3} n_{j}^{0} n_{k}^{0} \nabla_{j} \nabla_{k} n_{x} \\
\nabla_{j} v_{x j z y} & =\left(v_{3}-v_{2}\right)\left(n_{y}^{0} \nabla_{z} n_{x}+n_{z}^{0} \nabla_{y} n_{x}\right)+2\left(v_{1}+v_{2}-2 v_{3}\right) n_{y}^{0} n_{j}^{0} n_{z}^{0} \nabla_{j} n_{x} \\
v_{x j k l}^{0} \nabla_{j} \nabla_{k} v_{l} & =-v_{2} \Delta v_{x}-\left(v_{3}-v_{2}\right) n_{j}^{0} n_{k}^{0} \nabla_{j} \nabla_{k} v_{x}
\end{aligned}
$$

In (I. 5) we have assumed incompressibility, i.e. $v_{4}=v_{2}$ and $v_{5}=0$ for simplicity. The general case $v_{4} \neq v_{2}, v_{5} \neq 0$ can easily be obtained from [8]. For the special case $k_{x}=0$ and $\hat{n}^{0}=\hat{e}_{y} \cos \theta_{0}+\hat{e}_{z} \sin \theta_{0}$ equations (2.5) and (2.6) follow from (I.5). 
Appendix II. - In this appendix we will show that the $S_{1} z$-terms of equations (2.5) and (2.6) do not contribute to $\left\langle n_{x}(k \omega) n_{x}^{*}(k \omega)\right\rangle$ in linear order. We replace $z$ by $q^{-1} \sin q z$ with $q z \ll 1$ and $\mathbf{q}=q \hat{e}_{z}$. Fourier transforming equations (2.5) and (2.6) yields

$$
\begin{gathered}
\left(i \omega+\frac{i}{2} S_{0} d k_{y}+\xi \hat{K}_{\mathbf{k}}^{2}+\frac{1}{2} \Gamma\right) n_{\mathbf{k}}-\frac{i}{2}(\lambda+1) k_{\|} v_{\mathbf{k}}-\pi_{\mathbf{k}}=-\frac{S_{1} k_{y}}{2 q}\left(n_{\mathbf{k}-\mathbf{q}}-n_{\mathbf{k}+\mathbf{q}}\right) \\
\left(i \omega+\frac{i}{2} S_{0} d k_{y}+\hat{\mu}_{\mathbf{k}}^{2}\right) v_{\mathbf{k}}-\left(\frac{i}{2 \rho_{0}}[\lambda+1] k_{\|} \hat{K}_{\mathbf{k}}^{2}+i \hat{\alpha}_{\mathbf{k}} S_{1}\right) n_{\mathbf{k}}-\frac{i}{\rho_{0}} \mathbf{k} \cdot \sigma_{\mathbf{k}}= \\
=-\frac{S_{1} k_{y}}{2 q}\left(v_{\mathbf{k}-\mathbf{q}}-v_{\mathbf{k}+\mathbf{q}}\right) .
\end{gathered}
$$

Throughout this appendix we suppress the index $x$ on $n_{x}, v_{x}, \pi_{x}$ or $\sigma_{x i}$; therefore, $n_{\mathbf{k}-\mathbf{q}}$ is a short hand notation for $n_{x}\left(k_{\|}-q \sin \theta_{0}, k_{\perp}+q \cos \theta_{0}, \omega\right)$ etc. In zeroth order, i.e. omitting the r.h.s. of equations (II . 1), one can solve this inhomogeneous system of equations obtaining

$$
\begin{aligned}
& n_{\mathbf{k}}^{0}=A_{\mathbf{k}} \pi_{\mathbf{k}}+B_{\mathbf{k}} \sigma_{\mathbf{k}} \cdot \mathbf{k} \\
& v_{\mathbf{k}}^{0}=C_{\mathbf{k}} \sigma_{\mathbf{k}} \cdot \mathbf{k}+D_{\mathbf{k}} \pi_{\mathbf{k}}
\end{aligned}
$$

where the specific form of $A_{\mathbf{k}} \ldots D_{\mathbf{k}}$ is not needed here. Inserting the zero-order-solution (II.2) into the r.h.s. of (II . 1) leads to the first order result

$$
n_{\mathbf{k}}^{1}=A_{\mathbf{k}}\left[\pi_{\mathbf{k}}-\delta \pi_{\mathbf{k}-\mathbf{q}}+\delta \pi_{\mathbf{k}+\mathbf{q}}\right]+B_{\mathbf{k}}\left[\mathbf{k} \cdot \sigma_{k}+\delta \sigma_{\mathbf{k}-\mathbf{q}}-\delta \sigma_{\mathbf{k}+\mathbf{q}}\right]
$$

with

$$
\begin{aligned}
& \delta \pi_{\mathbf{k} \pm \mathbf{q}}=\frac{S_{1} k_{y}}{2 q}\left[A_{\mathbf{k} \pm \mathbf{q}} \pi_{\mathbf{k} \pm \mathbf{q}}+B_{\mathbf{k} \pm \mathbf{q}} \sigma_{\mathbf{k} \pm \mathbf{q}} \cdot(\mathbf{k} \pm \mathbf{q})\right] \\
& \delta \sigma_{\mathbf{k} \pm \mathbf{q}}=i \frac{S_{1} k_{y} \rho_{0}}{2 q}\left[C_{\mathbf{k} \pm \mathbf{q}} \sigma_{\mathbf{k} \pm \mathbf{q}}(\mathbf{k} \pm \mathbf{q})+D_{\mathbf{k} \pm \mathbf{q}} \pi_{\mathbf{k} \pm \mathbf{q}}\right]
\end{aligned}
$$

The required correlation function is given in first order by

$$
\begin{aligned}
\left\langle n_{\mathbf{k}}^{1} n_{\mathbf{k}}^{1 *}\right\rangle-\left\langle n_{\mathbf{k}}^{0} n_{\mathbf{k}}^{0 *}\right\rangle= & -\left|A_{\mathbf{k}}\right|^{2}\left\langle\pi_{\mathbf{k}} \delta \pi^{*}+\pi_{\mathbf{k}}^{*} \delta \pi\right\rangle \\
& -B_{\mathbf{k}} A_{\mathbf{k}}^{*}\left\langle\delta \sigma \pi_{\mathbf{k}}^{*}+\mathbf{k} . \sigma_{\mathbf{k}} \delta \pi^{*}\right\rangle \\
& -B_{\mathbf{k}}^{*} A_{\mathbf{k}}\left\langle\delta \sigma^{*} \pi_{\mathbf{k}}+\mathbf{k} \cdot \sigma_{\mathbf{k}}^{*} \delta \pi\right\rangle \\
& -\left|B_{\mathbf{k}}\right|^{2}\left\langle\mathbf{k} . \sigma_{\mathbf{k}} \delta \sigma^{*}+\mathbf{k} . \sigma_{\mathbf{k}}^{*} \delta \sigma\right\rangle .
\end{aligned}
$$

However, the r.h.s. of (II .4) vanishes identically, since $\delta \sigma$ and $\delta \pi$ contain only fluctuating forces at wave vectors $\mathbf{k} \pm \mathbf{q}$, while $\sigma_{\mathbf{k}}$ and $\pi_{\mathbf{k}}$ are at wave vectors $\mathbf{k}$ and $\left\langle\pi_{\mathbf{k}} \pi_{\mathbf{k}^{\prime}}^{*}\right\rangle=\left\langle\pi_{\mathbf{k}} \sigma_{\mathbf{k}^{\prime}}\right\rangle=$ $\left\langle\sigma_{\mathbf{k}} \sigma_{\mathbf{k}^{\prime}}^{*}\right\rangle=0$ for $\mathbf{k} \neq \mathbf{k}^{\prime}$ according to (2.8). Therefore, in first order, $\left\langle n_{\mathbf{k}} n_{\mathbf{k}}^{*}\right\rangle$ is the same, as we had omitted the $S_{1} z$-terms from the beginning. 


\section{References}

[1] Procaccia, I., Ronis, D. and Oppenheim, I., Phys. Rev. Lett. 42 (1979) 287.

[2] Kirkpatrick, T., Cohen, E. G. D. and Dorfman, J. R., Phys. Rev. Lett. 42 (1979) 862.

[3] Tremblay, A. M. S., Siggia, E. D. and Arai, M. R., Phys. Lett. A 76 (1980) 57.

Tremblay, A. M. S., Arai, M. and Siggia, E. D., Phys. Rev. A 23 (1981) 1451.

[4] Keyes, T., Phys. Rev. A 23 (1981) 277.

[5] Beysens, D., Garrabos, Y. and Zalczer, V., Phys. Rev. Lett. 45 (1980) 403.

[6] Pleiner, H. and Brand, H., Phys. Rev. A 26, in press.

[7] Forster, D., Phys. Rev. Lett. 32 (1974) 1161.

[8] De Gennes, P. G., Physics of Liquid Crystals (Clarendon, Oxford) 1974.

[9] Forster, D., Hydrodynamic Fluctuations, Broken Symmetry and Correlation Functions (Benjamin, Reading, Mass) 1975.

[10] Landau, L. D. and Lifshitz, E. M., Statistical Physics (Addison-Wesley, Reading Mass.) 1969.

[11] Pieranski, P. and Guyon, E., Phys. Rev. Lett. 32 (1974) 924.

[12] Pieranski, P. and Guyon, E., Phys. Rev. A 9 (1974) 404.

[13] Manneville, P. and Dubois-Violette, E., J. Physique 37 (1976) 285.

[14] Dubois-Violette, E., Guyon, E., Janossy, I., Pieranski, P. and Mannevilile, P., J. Méc. 16 (1977) 733.

[15] Dubois-Violette, E., Durand, G., Guyon, E., Manneville, P. and Pieranski, P., in Solid State Physics : advances in Research and Applications, Supplement 14, L. Liebert Ed. (Academic Press) 1978.

[16] Pieranski, P. and Guyon, E., Comm. Phys. 1 (1976) 45.

[17] ZwanZIG, R., in Statistical Physics edited by S. Rice, K. Freed and J. Light (University of Chicago Press, Chicago) 1972.

[18] Brand, H. and Pleiner, H., J. Physique 41 (1980) 553. 